\title{
New Results with High Quantum Efficiency Silicon Drift Detectors
}

\author{
A.Niculae*, L.Andricek***, M.Bornschlegl*, R.Eckhardt**, J.Herrmann*, O.Jaritschin*, \\ S.Jeschke**, P.Lechner**, L.Mungenast**, B.Schweinfest**, H.Soltau**, L.Strüder*** \\ *PNDetector GmbH, Emil-Nolde-Str.10, D-81735 München, Germany \\ **PNSensor GmbH, Römerstr. 28, D-80803 München, Germany \\ ***MPI Halbleiterlabor, Otto-Hahn-Ring 6, D-81739 München, Germany
}

Silicon Drift Detectors (SDD) are nowadays very popular for a large variety of instruments in the field of X-ray Fluorescence Spectroscopy (XRF). Their advantage of having a very small input capacitance which is independent on the detector active area makes them indispensable for applications requiring good energy resolution at high input count rates.

The SDD detectors manufactured by the companies PNDetector and PNSensor in Munich combine the advantage of the SDD principle with the monolithical integration of the first amplification FET directly onto the detector chip. Thus, the total input capacitance is kept free of large interconnection capacitances and by that, its noise contribution to the detector is reduced to minimum levels especially at high count rates. Furthermore, the very well controlled fabrication technology which is entirely dedicated to detector fabrication leads to very low levels of detector leakage current and therefore greatly reducing the effort for the detector cooling. This becomes more significant when considering the general trend of moving towards detectors with larger sensitive area.

Recently we developed new types of SDD detector modules combining the large detector area with application specific detector geometry. One of the detectors presented here has a large sensitive area of $60 \mathrm{~mm}^{2}$ with a round shaped sensor. Mounted into a compact package (see picture in Fig 1), the detector is dedicated to EDS applications where a large collection solid angle is needed. The other detector which is discussed here has an active area of $100 \mathrm{~mm}^{2}$ with the shape of a rounded rectangle (see picture in Fig 2). The detector can be employed as a large area detector for EDX as well as WDX spectrometers. Spectroscopic results with both detectors will be shown and the performance values will be discussed.

Besides the geometry of the sensor chip, the packaging of the detector unit is also important for the application. This includes the optimization of the package geometry, the careful choice of the materials used for the mounting parts with regard to magnetization or trace contamination, as well as the radiation entrance window of the detector. We will present results with detector configurations optimized with regards to the package geometry, background fluorescence peaks and radiation entrance window. 


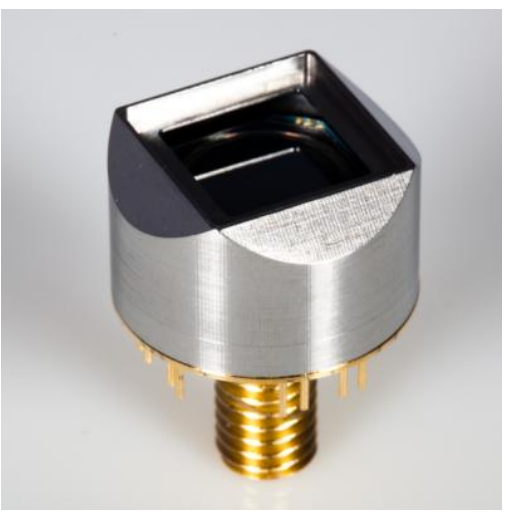

Fig. 1. SDD module with $60 \mathrm{~mm}^{2}$ active area

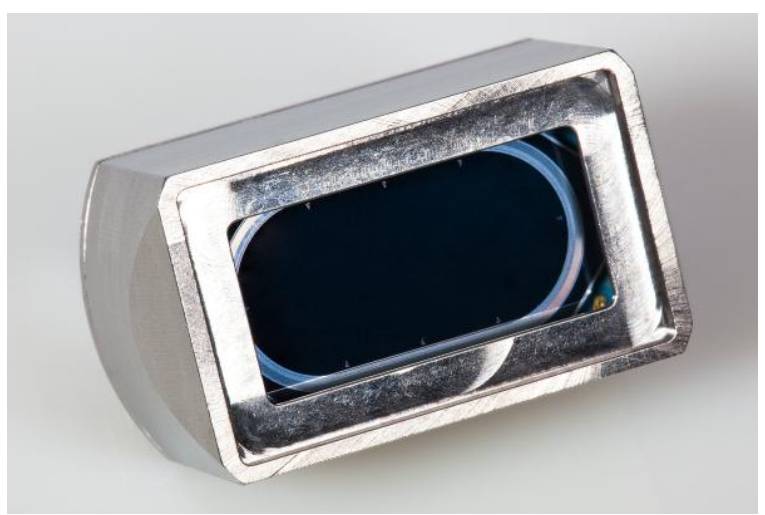

Fig. 2 SDD with $100 \mathrm{~mm}^{2}$ active area and oval shape

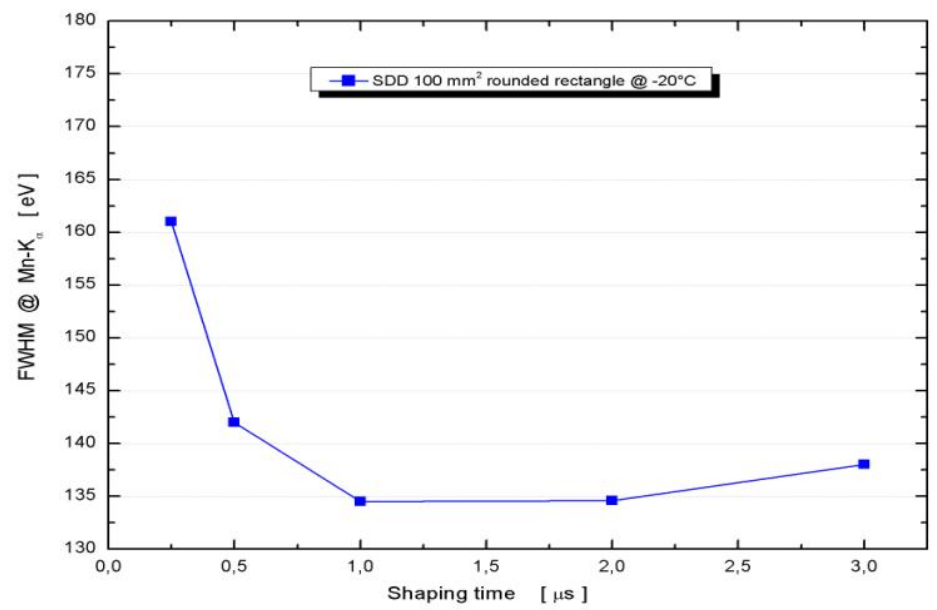

Fig. 3 Energy resolution of a $100 \mathrm{~mm}^{2}$ oval shaped detector measured at different shaping times 